\title{
Diffusional Kurtosis Imaging and Motor Outcome in Acute Ischemic Stroke
}

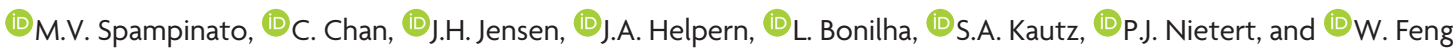

\begin{abstract}
BACKGROUND AND PURPOSE: Motor impairment is the most common deficit after stroke. Our aim was to evaluate whether diffusional kurtosis imaging can detect corticospinal tract microstructural changes in the acute phase for patients with first-ever ischemic stroke and motor impairment and to assess the correlations between diffusional kurtosis imaging-derived diffusion metrics for the corticospinal tract and motor impairment 3 months poststroke.
\end{abstract}

MATERIALS AND METHODS: We evaluated 17 patients with stroke who underwent brain MR imaging including diffusional kurtosis imaging within 4 days after the onset of symptoms. Neurologic evaluation included the Fugl-Meyer Upper Extremity Motor scale in the acute phase and 3 months poststroke. For the corticospinal tract in the lesioned and contralateral hemispheres, we estimated with diffusional kurtosis imaging both pure diffusion metrics, such as the mean diffusivity and mean kurtosis, and model-dependent quantities, such as the axonal water fraction. We evaluated the correlations between corticospinal tract diffusion metrics and the Fugl-Meyer Upper Extremity Motor scale at 3 months.

RESULTS: Among all the diffusion metrics, the largest percentage signal changes of the lesioned hemisphere corticospinal tract were observed with axial kurtosis, with an average $12 \%$ increase compared with the contralateral corticospinal tract. The strongest associations between the 3-month Fugl-Meyer Upper Extremity Motor scale score and diffusion metrics were found for the lesioned/contralateral hemisphere corticospinal tract mean kurtosis $(\rho=-0.85)$ and axial kurtosis $(\rho=-0.78)$ ratios.

CONCLUSIONS: This study was designed to be one of hypothesis generation. Diffusion metrics related to kurtosis were found to be more sensitive than conventional diffusivity metrics to early poststroke corticospinal tract microstructural changes and may have potential value in the prediction of motor impairment at 3 months.

ABBREVIATIONS: CST = corticospinal tract; $\mathrm{D}_{\mathrm{a}}=$ intra-axonal diffusivity; $\mathrm{DKI}=$ diffusional kurtosis imaging; $\mathrm{FA}=$ fractional anisotropy; FM-UE $=$ Fugl-Meyer Upper Extremity Motor; $\mathrm{K}_{\|}=$axial kurtosis; $\mathrm{K}_{\perp}=$ radial kurtosis; $\lambda_{\|}=$axial diffusivity; $\lambda_{\perp}=$ radial diffusivity; $\mathrm{MD}=$ mean diffusivity; $\mathrm{MK}=$ mean kurtosis

M otor impairment caused by injury of the motor pathways is the most common deficit after stroke. ${ }^{1}$ While a proportion of patients with acute stroke with motor deficits will have func-

Received August 8, 2016; accepted after revision February 13, 2017.

From the Department of Radiology and Radiological Science (M.V.S., C.C., J.H.J., J.A.H.), Center for Biomedical Imaging (M.V.S., C.C., J.H.J., J.A.H.), Department of Health Sciences and Research (S.A.K.), Department of Public Health Sciences (P.J.N., W.F.), and Department of Neurology (L.B., W.F., J.A.H.), Medical University of South Carolina, Charleston, South Carolina; and Ralph H. Johnson VA Medical Center (S.A.K.), Charleston, South Carolina.

This work was supported, in part, by the South Carolina Clinical and Translational Research Institute at the Medical University of South Carolina, funded by the $\mathrm{Na}$ tional Institutes of Health, National Center for Advancing Translational Science (grant No. ULITR001450). Dr Feng was supported by American Heart Association (14SDG1829003) and Drs Feng, Bonilha, and Kautz were supported by National Institute of Health (P20 GM109040).

Please address correspondence to Maria Vittoria Spampinato, MD, Department of Radiology and Radiological Science, Medical University of South Carolina, 96 Jonathan Lucas, MSC 323, Charleston, SC 29425; e-mail: spampin@musc.edu; (a)vspampinato tional recovery, the degree of motor recovery is highly variable. ${ }^{2}$ Clinical assessment of motor impairment in the acute phase is a significant predictor of chronic motor outcome. ${ }^{3}$ However, for patients with severe initial motor impairment, long-term motor outcome is only weakly predicted by the degree of motor impairment in the acute phase. ${ }^{4}$ Thus, it remains difficult to make an accurate motor outcome prediction for an individual patient with information collected in the acute phase. If clinicians had access to an early marker of motor pathway injury and were able to predict motor outcome, stroke recovery could be greatly improved by delivering treatment to those individuals who are likely to benefit from intervention.

The integrity of the corticospinal tract (CST) is critical for

\footnotetext{
-- Indicates open access to non-subscribers at www.ajnr.org

三 Indicates article with supplemental on-line table.

http://dx.doi.org/10.3174/ajnr.A5180
} 
recovery of motor function in stroke. ${ }^{5}$ Identification of early CST microstructural changes in patients with acute stroke would improve our ability to predict motor recovery and plan rehabilitative treatment. DTI can quantify early CST microstructural changes in patients with stroke with motor impairment. ${ }^{6-10}$ However, a limitation of DTI is that the data analysis approximates the water diffusion dynamics within brain tissue as being a Gaussian process, though substantial non-Gaussian diffusion effects are observed throughout the brain. Hence, DTI does not fully characterize water diffusion in the brain. Diffusional kurtosis imaging (DKI) is a clinically feasible diffusion MR imaging method, which extends the DTI model to include non-Gaussian diffusion effects. ${ }^{11,12}$ As a result, DKI has the potential to provide more sensitive biomarkers for probing microscopic structural changes. ${ }^{13}$ In addition, white matter tract integrity metrics can be obtained from the DKI dataset by using a model that describes the microstructural characteristics in the extra- and intra-axonal compartments of WM. ${ }^{14}$ To our knowledge, no prospective studies have previously assessed the role of DKI in the prediction of motor outcome after stroke.

Our objectives were to evaluate whether DKI-derived diffusion metrics can detect early CST microstructural changes 1-4 days after first-ever acute ischemic stroke and motor impairment and to assess the correlations between CST DKI-derived metrics and 3-month motor outcome measured by using the Fugl-Meyer Upper Extremity Motor (FM-UE) scale.

\section{MATERIALS AND METHODS \\ Subjects}

The local institutional review board approved the study. Patients with first-ever ischemic stroke with various degrees of motor impairment were enrolled in this prospective observational study. Written informed consent was obtained from all patients or their legal representatives. Inclusion criteria were the following: 18-80 years of age; first-ever ischemic stroke involving only 1 hemisphere, confirmed by MR imaging; brain MR imaging, including DKI, performed between 1 and 4 days after stroke onset; prestroke mRS $\leq 1$; and unilateral motor impairment at baseline with an FM-UE scale score of $\leq 56$ of 66 (to avoid recovery ceiling effect). We excluded any subject with intracranial hemorrhage, other neurologic disorders affecting limb motor function, severe dementia, recurrent stroke, or death during the 3-month follow-up.

A detailed neurologic examination was performed by a stroke neurologist in the acute phase and at a 3-month follow-up visit. The baseline examination included the FM-UE scale and the NIHSS (Table 1). During the 3-month follow-up examination, the FM-UE scale, NIHSS, and mRS scores were obtained. The FM-UE scale, a motor assessment scale with excellent intra- and interrater reliability, represented the primary motor outcome variable (the maximum score is 66 points; higher scores indicate less severe impairment). ${ }^{15}$ We also calculated the percentage change of the FM-UE scale between the baseline and 3-month evaluation. For descriptive purposes, patients were divided into 3 cohorts based on the 3-month FM-UE scale: 1) upper limb remained essentially plegic (FM-UE $\leq 9) ; 2$ ) patients with welldefined upper limb mass flexion and extension synergy-dependent movements (FM-UE $=10-18$ ); and 3) patients with
Table 1: Patient clinical characteristics and demographics $(N=17)$ Demographics and Characteristics

\begin{tabular}{|c|c|}
\hline Age $(y r)^{a}$ & $55.7(12.3)$ \\
\hline Men & $64.7 \%$ \\
\hline Race (white/African American) & $12 / 5$ \\
\hline Lesion side (right) & $70.6 \%$ \\
\hline \multicolumn{2}{|l|}{ Stroke etiology } \\
\hline Small-vessel disease & $35.3 \%$ \\
\hline Cardioembolism & $17.6 \%$ \\
\hline Large-vessel atherosclerosis & $29.4 \%$ \\
\hline Unknown & $17.6 \%$ \\
\hline rtPA or reperfusion therapy & $41.2 \%$ \\
\hline Hypertension & $82.4 \%$ \\
\hline Diabetes mellitus & $35.3 \%$ \\
\hline Dyslipidemia & $70.6 \%$ \\
\hline Atrial fibrillation & $23.5 \%$ \\
\hline Coronary artery disease & $11.8 \%$ \\
\hline Antiplatelet use & $88.2 \%$ \\
\hline Anticoagulation use & $17.6 \%$ \\
\hline Statin on discharge & $82.3 \%$ \\
\hline Smoking on admission & $47.1 \%$ \\
\hline Length of hospital stay (days) ${ }^{a}$ & $5.8(3.8)$ \\
\hline \multicolumn{2}{|l|}{ Clinical assessment } \\
\hline Days between stroke onset and MRI ${ }^{\mathrm{a}}$ & $2.0(1.0)$ \\
\hline Days from stroke onset to baseline assessment $t^{a}$ & $2.6(1.5)$ \\
\hline Days from admission to follow-up visit ${ }^{a}$ & $94.1(9.6)$ \\
\hline Median No. of days of rehabilitation therapy & 34 (18.1) \\
\hline Baseline NIHSS score ${ }^{a}$ & $11.2(6.5)$ \\
\hline 3-mo NIHSS score ${ }^{a}$ & $4.9(3.9)$ \\
\hline Baseline FM-UE scale ${ }^{a}$ & $19.1(16.9)$ \\
\hline 3-month FM-UE scale ${ }^{a}$ & $29.1(22.0)$ \\
\hline Median 3-mo mRS (range) & $3(1-4)$ \\
\hline \multicolumn{2}{|l|}{ Location of infarction (No.) (\%) } \\
\hline Right hemisphere & $12(70.5)$ \\
\hline Left hemisphere & $5(29.5)$ \\
\hline Motor cortex & $6(35.3)$ \\
\hline Premotor cortex & $5(29.5)$ \\
\hline Centrum semiovale & $4(23.5)$ \\
\hline Corona radiata & $9(52.9)$ \\
\hline Posterior limb of the internal capsule & $9(52.9)$ \\
\hline Brain stem & $4(23.5)$ \\
\hline
\end{tabular}

${ }^{a}$ Mean (SD).

recovery of isolated upper limb movements (FM-UE $\geq 19) .{ }^{16} \mathrm{We}$ also recorded the total number of days each patient received rehabilitative therapy (including physical therapy and/or occupational therapy during rehabilitation, at home, and during outpatient visits) between the stroke and the 3-month follow-up.

\section{Imaging}

Brain MRIs were performed on a 1.5T MR imaging scanner. A sagittal T1-weighted MPRAGE (voxel size, $1 \times 1 \times 1 \mathrm{~mm}^{3}$ ) sequence was performed. Axial diffusion-weighted images were acquired with $3 \mathrm{~b}$-values $\left(0,1000\right.$, and $\left.2000 \mathrm{~s} / \mathrm{mm}^{2}\right)$ along $30 \mathrm{diffu}-$ sion-encoding directions by using a single-shot twice-refocused spin-echo EPI sequence with NEX $=1(\mathrm{NEX}=10$ for $b=0)$. Imaging parameters of the diffusion sequence were the following: voxel size $=3 \times 3 \times 3 \mathrm{~mm}^{3}$, number of sections $=40$, TR/TE $=$ $5500 / 99 \mathrm{~ms}, \mathrm{FOV}=222 \times 222 \mathrm{~mm}^{2}$, acquisition matrix $=74 \times$ 74 , bandwidth/pixel $=1325 \mathrm{~Hz}$, acceleration factor $=2$, acquisition time $\approx 7$ minutes.

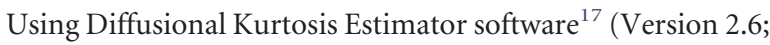
https://www.nitrc.org/projects/dke/) implemented in Matlab (MathWorks, Natick, Massachusetts), we calculated the diffusion 


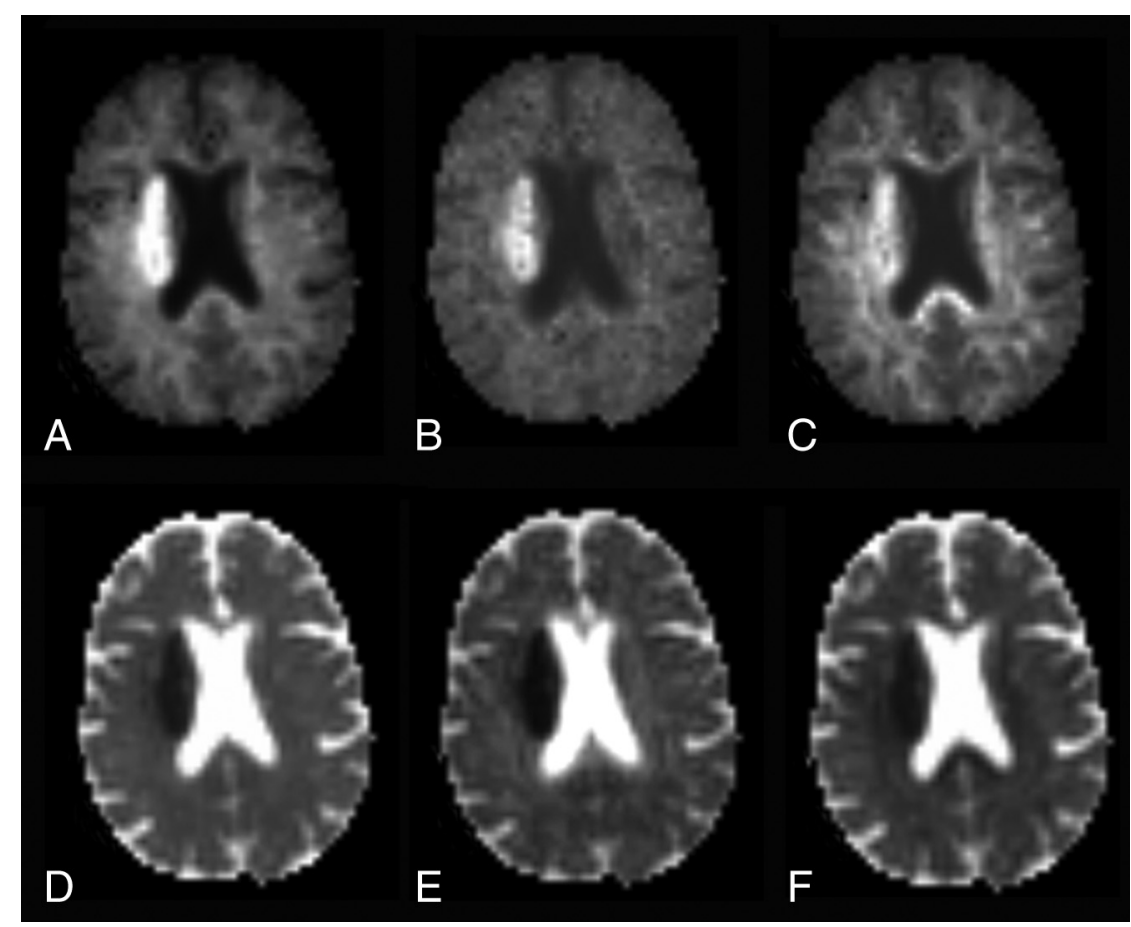

FIG 1. Mean kurtosis $(A)$, axial kurtosis $(B)$, radial kurtosis $(C)$, mean diffusivity $(D)$, axial diffusivity $(E)$, and radial diffusivity $(F)$ maps obtained from the DKI dataset in a representative patient with acute ischemic infarct of the right corona radiata.

and kurtosis tensors on a voxel-by-voxel basis. Parametric maps for the following metrics were obtained from the DKI dataset: 1) diffusivity metrics: fractional anisotropy (FA), mean diffusivity (MD), axial $\left(\lambda_{\|}\right)$, and radial diffusivity $\left.\left(\lambda_{\perp}\right) ; 2\right)$ kurtosis metrics: mean kurtosis $(\mathrm{MK})$, axial $\left(\mathrm{K}_{\|}\right)$, and radial kurtosis $\left(\mathrm{K}_{\perp}\right)($ Fig 1$),{ }^{11}$ in which $\mathrm{MK}$ is the average kurtosis over all diffusion directions, $\mathrm{K}_{\|}$is the kurtosis in the direction of the diffusion tensor eigenvector with the largest diffusion eigenvalue (typically along the direction of the axons), and $\mathrm{K}_{\perp}$ is the average kurtosis over all directions perpendicular to the diffusion eigenvector with the largest eigenvalue (typically all directions perpendicular to the axons); and 3) white matter tract integrity metrics: axonal water fraction, intra-axonal diffusivity $\left(\mathrm{D}_{\mathrm{a}}\right)$, axial extra-axonal diffusivity, and radial extra-axonal diffusivity. White matter tract integrity metrics were obtained from the DKI dataset in WM voxels consisting of aligned fiber bundles (defined here as FA $\geq 0.3$ ). ${ }^{14}$ In the white matter tract integrity model, axonal fiber bundles are idealized as impermeable, cylindric tubes, and the axonal water fraction represents the fraction of diffusion MR imaging-visible water contained within the tubes. Water within myelin is thus neglected because it contributes little to the diffusion MR imaging signal due to its short $\mathrm{T} 2$ relaxation time. Qualitatively, the axonal water fraction can be regarded as an index of axonal fiber density. $\mathrm{D}_{\mathrm{a}}$ is a measure of intrinsic diffusivity inside the axons. The remainder of the WM is modeled as the extra-axonal space, and axial extraaxonal diffusivity and radial extra-axonal diffusivity are markers of changes in extra-axonal diffusivity.

Fiber tracking of the CST was performed by using DSI Studio (http://dsi-studio.labsolver.org) with a generalized deterministic tracking algorithm that uses quantitative anisotropy as the termination index. ${ }^{18}$ The CST was reconstructed as previously de- scribed ${ }^{19}$ in accordance with the following steps: The tractography seed point was obtained by manually placing an ROI on each CST at the level of the rostral pons on the color-coded FA maps, and the tractography end point was obtained by manually placing a gray matter ROI on the ipsilateral precentral gyrus (anisotropy threshold $=0.2$; angular threshold $=40^{\circ}$; step size $=1 \mathrm{~mm}$; track length $=\geq 50 \mathrm{~mm}$; number of seeds $=$ 10,000). The lesioned hemisphere CST could be reliably identified at the rostral pons in all subjects, even in patients with large infarctions. We added a logical "and" function so that only streamlines passing through both ROIs were included for further analysis. Fiber tracts were generated of the left and right CST. A neuroradiologist, blinded to demographic information and clinical data, visually inspected the CST tracts overlaid on the FA map for quality control purposes. Then, left and right CST tracts were converted to VOIs in native diffusion space. Average diffusion values of the CST VOIs were computed for all diffusion metrics and subjects by using Matlab. Lesioned hemisphere/contralateral hemisphere CST ratios were computed for all diffusion metrics.

Multisection VOIs of the infarct were identified by a neuroradiologist, blinded to demographic and clinical information, on the diffusion-weighted images with b-values of 2000 $\mathrm{s} / \mathrm{mm}^{2}$ with the intensity filter module of MRICron (http:// www.mccauslandcenter.sc.edu/crnl/mricron/). The volumes of the infarct VOI, ipsilateral CST VOI, and the intersection between the CST and infarct VOIs were calculated by using MRICron.

Statistical analyses were performed by using SPSS, Version 22 (IBM, Armonk, New York). Comparisons of the ipsilateral and contralateral CST diffusion metrics were performed by using the paired Wilcoxon signed rank test. Associations between average CST diffusion metrics and the 3-month FM-UE scale were evaluated by using Spearman rank correlation tests. Results were considered significant with $P<.05$. Because this study was designed largely to be one of hypothesis generation, $P$ values were not corrected for multiple comparisons.

\section{RESULTS}

\section{Clinical Data}

Twenty-one patients were recruited for this study. Subsequently, 3 patients were excluded because they were lost to follow-up and 3-month follow-up data were not available. One patient was excluded because the DKI dataset was degraded by artifacts from bulk motion. Hence, 17 patients were included in the analysis (Table 1). All patients received rehabilitation therapy (median number of days of therapy $=34 \pm 18$ ). Overall, the FM-UE scale score significantly improved between the baseline and the 3-month neurologic examination $(P=.001)$, with motor func- 
Table 2: Diffusion metrics of lesioned and contralateral corticospinal tract in stroke with motor impairment

\begin{tabular}{|c|c|c|c|}
\hline Diffusion Metric & $\begin{array}{l}\text { Lesioned } \\
\text { CST }^{\mathrm{a}}\end{array}$ & $\begin{array}{c}\text { Contralateral } \\
\text { CST }^{\mathrm{a}}\end{array}$ & $P$ Value \\
\hline MD & $0.91(0.07)$ & $0.96(0.03)$ & .003 \\
\hline Axial diffusivity & $1.35(0.12)$ & $1.44(0.06)$ & .002 \\
\hline Radial diffusivity & $0.70(0.05)$ & $0.72(0.04)$ & .031 \\
\hline Fractional anisotropy & $0.41(0.03)$ & $0.43(0.03)$ & .025 \\
\hline MK & $1.19(0.12)$ & $1.10(0.07)$ & .002 \\
\hline Axial kurtosis & $0.94(0.11)$ & $0.84(0.03)$ & .001 \\
\hline Radial kurtosis & $1.48(0.18)$ & $1.43(1.13)$ & .193 \\
\hline AWF & $0.41(0.02)$ & $0.40(0.02)$ & .246 \\
\hline Intra-axonal diffusivity & $0.71(0.09)$ & $0.78(0.06)$ & .001 \\
\hline Extra-axonal axial diffusivity & $1.95(0.17)$ & $2.05(0.10)$ & .002 \\
\hline Extra-axonal radial diffusivity & $1.07(0.07)$ & $1.10(0.05)$ & .049 \\
\hline
\end{tabular}

Note:-AWF indicates axial water fraction.

${ }^{a}$ Mean (SD).

tion improvement in 14 subjects, no changes in 2 subjects, and worsening function in 1 subject. At 3 months, 3 patients had little if any upper extremity motor function; 5 patients had well-defined upper limb mass flexion and extension synergy-dependent movements; and 9 patients had isolated upper limb movements. There was no significant correlation between the number of days of rehabilitation therapy and the baseline FM-UE scale score ( $r=-0.340, P=.181)$ or the 3 -month FM-UE scale score $(r=$ $-0.373, P=.140)$.

\section{Imaging Data}

Please see Table 1 for details regarding infarct location and Table 2 for CST diffusion metrics. CST MD, FA, $\lambda_{\|}, \lambda_{\perp}, \mathrm{D}_{\mathrm{a}}$, axial extraaxonal diffusivity, and radial extra-axonal diffusivity were lower in the lesioned than in the contralateral hemisphere. MK and $\mathrm{K}_{\|}$ were greater in the lesioned than in the contralateral hemisphere CST. The On-line Table demonstrates infarct volume, CST volume, their intersection, and diffusion metrics for the entire cohort and for patients stratified on the basis of the degree of recovery at 3 months. There was a strong association between baseline and 3 -month FM-UE scale $(\rho=0.8, P=.001)$. We found significant correlations among the 3-month FM-UE scale, percentage change of FM-UE scale between the baseline and 3-month visit, diffusion metrics of the lesioned hemisphere, and lesioned/contralateral hemisphere CST ratios (Table 3). The strongest associations were found between the 3-month FM-UE scale score and MK, and the 3-month FM-UE scale score and $\mathrm{K}_{\|}$, among kurtosis metrics; and between the 3-month FM-UE scale score and MD, among diffusivity metrics (Fig 2). We did not find any significant correlation between the number of days of rehabilitation therapy and kurtosis metrics. For example, there was no significant association between the number of days of rehabilitation therapy and lesional/contralateral MK $(\rho=0.212, P=.398), \mathrm{K}_{\|}(\rho=0.282$, $P=.257), \operatorname{MD}(\rho=-0.318, P=.198), \lambda_{\|}(\rho=-0.330, P=$ $.181)$, and FA $(\rho=-0.78, P=.759)$.

\section{DISCUSSION}

We conducted a prospective observational study designed to explore the hypothesis that DKI-derived metrics can be useful in assessing CST microstructural changes and predicting motor outcome after stroke. Due to the relatively small sample size, this proof-of-concept study is mainly for hypothesis generation, and
Table 3: Correlations among the baseline FM-UE scale score, corticospinal tract diffusion metrics, and 3-month FM-UE scale score

\begin{tabular}{|c|c|c|c|c|}
\hline & \multicolumn{2}{|c|}{$\begin{array}{l}\text { Lesioned } \\
\text { Hemisphere } \\
\text { CST }\end{array}$} & \multicolumn{2}{|c|}{$\begin{array}{l}\text { Lesioned/ } \\
\text { Contralateral } \\
\text { CST Ratio }\end{array}$} \\
\hline & $\rho$ & $P$ Value & $\boldsymbol{\rho}$ & $P$ Value \\
\hline \multicolumn{5}{|l|}{ 3-mo FM-UE scale } \\
\hline MD & 0.65 & .007 & 0.69 & .002 \\
\hline Axial diffusivity & 0.55 & .022 & 0.73 & .001 \\
\hline Radial diffusivity & 0.53 & .030 & 0.60 & .011 \\
\hline FA & 0.08 & .761 & 0.40 & .111 \\
\hline MK & -0.72 & .001 & -0.85 & $<.001$ \\
\hline Axial kurtosis & -0.75 & .001 & -0.78 & $<.001$ \\
\hline Radial kurtosis & -0.33 & 196 & -0.47 & .056 \\
\hline AWF & -0.45 & .072 & -0.63 & .007 \\
\hline Intra-axonal diffusivity & 0.51 & .035 & 0.48 & .054 \\
\hline Axial extra-axonal diffusivity & 0.45 & .070 & 0.61 & .009 \\
\hline Radial extra-axonal diffusivity & 0.37 & .142 & 0.45 & .072 \\
\hline \multicolumn{5}{|l|}{ Change of FM-UE scale (\%) } \\
\hline MD & 0.64 & .006 & 0.47 & .055 \\
\hline Axial diffusivity & 0.49 & .046 & 0.66 & .004 \\
\hline Radial diffusivity & 0.69 & .002 & 0.42 & .098 \\
\hline FA & -0.01 & .974 & 0.47 & .060 \\
\hline MK & -0.61 & .010 & -0.72 & .001 \\
\hline Axial kurtosis & -0.65 & .005 & -0.65 & .004 \\
\hline Radial kurtosis & -0.28 & .269 & -0.48 & .049 \\
\hline AWF & -0.54 & .026 & -0.59 & .013 \\
\hline Intra-axonal diffusivity & 0.42 & .095 & 0.43 & .083 \\
\hline Axial extra-axonal diffusivity & 0.42 & .090 & 0.56 & .019 \\
\hline Radial extra-axonal diffusivity & 0.53 & .028 & 0.24 & .349 \\
\hline
\end{tabular}

Note:-AWF indicates axial water fraction.

our results will require validation in future studies with larger sample sizes. Both diffusivity and kurtosis diffusion metrics could detect early microstructural changes of the CST in the acute poststroke phase, with larger differences between the ipsilateral and contralateral CST being observed for the kurtosis metrics. We also found moderately strong associations between motor outcome with the 3-month FM-UE scale and several diffusion metrics. The strongest motor outcome correlations were with $\mathrm{MK}(\rho=-0.72)$ and $\mathrm{K}_{\|}(\rho=-0.75)$ of the lesioned CST, and with the lesioned/ contralateral hemisphere $\operatorname{CST} \operatorname{MK}(\rho=-0.85)$ and $\mathrm{K}_{\|}(\rho=$ $-0.78)$ ratios.

The observation of early CST diffusion changes is consistent with the known dynamics of acute neuronal damage after stroke, a phenomenon known as Wallerian degeneration. ${ }^{8}$ In agreement with prior studies, we found that diffusivity metrics differed between the lesioned hemisphere CST and the contralateral CST, ${ }^{20}$ with greater acute-early subacute changes in $\lambda_{\|}$and MD compared with $\lambda_{\perp} \cdot{ }^{21}$ Kurtosis metrics, specifically $\mathrm{MK}, \mathrm{K}_{\|}, \mathrm{D}_{\mathrm{a}}$, and axial extra-axonal diffusivity, could identify early microstructural changes of the CST. The kurtosis parameters provide microstructural information not available by using conventional DTI, ${ }^{11}$ and it is important to assess whether the enhanced sensitivity to microstructural changes provided by DKI translates into improved motor outcome prediction. Larger absolute percentage changes for kurtosis metrics than for diffusivity metrics have been consistently reported in ischemic stroke in the animal and human literature. ${ }^{13,22,23}$ Here, we find that kurtosis metrics also have greater sensitivity to the detection of CST microstructural alterations, as reflected by their larger absolute percentage changes. For example, we found an average $12 \%$ increase in $\mathrm{K}_{\|}$and an average $6.5 \%$ decrease in $\lambda_{\|}$of the ipsilateral compared with the contralateral 

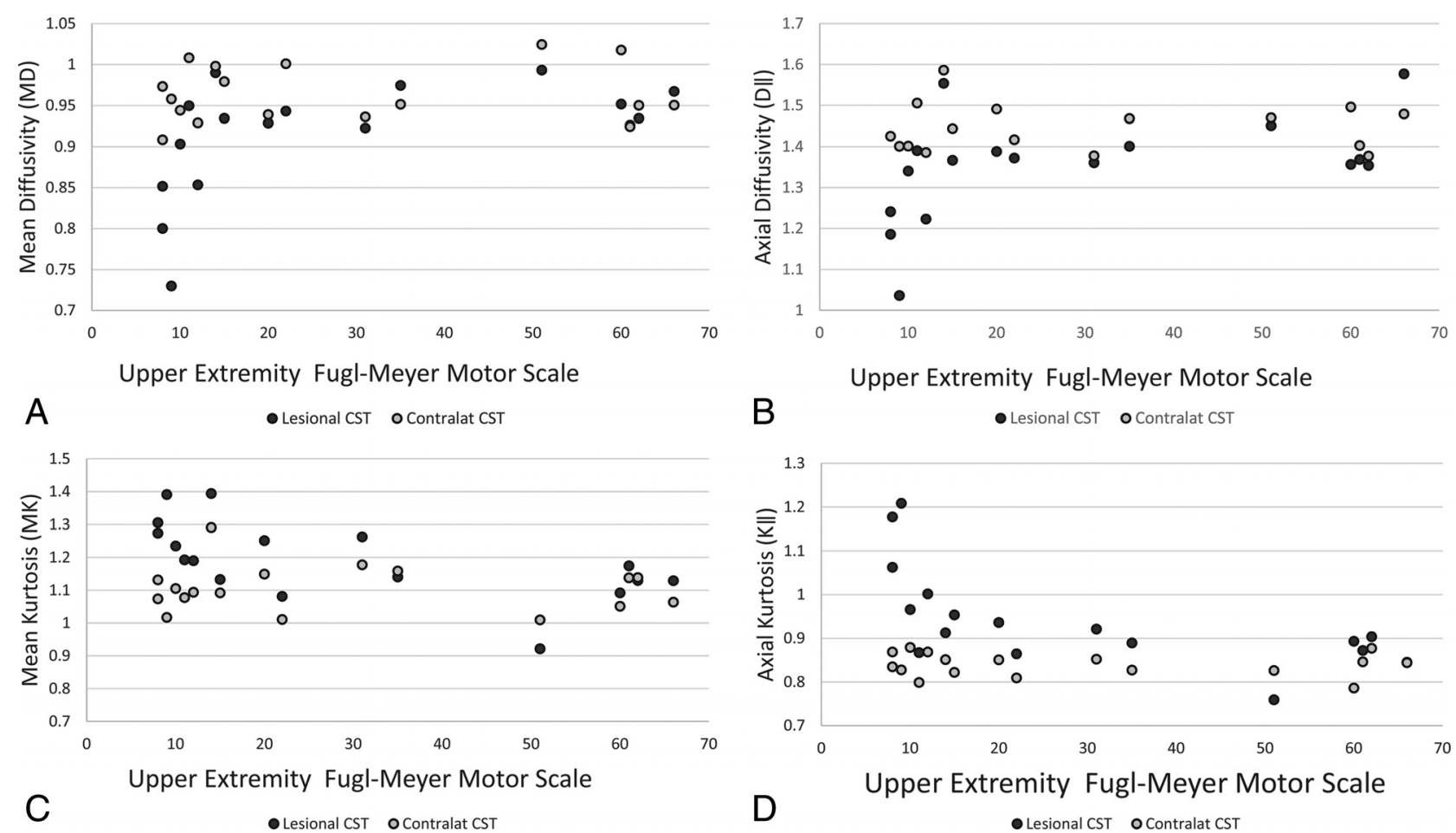

FIG 2. Scatterplots for the Upper Extremity Fugl-Meyer Motor scale versus mean diffusivity $(A)$, axial diffusivity (B), mean kurtosis (C), and axial kurtosis $(D)$ of the corticospinal tracts.

CST. Absolute percentage changes were larger along the axial direction than along the radial direction of the CST WM. ${ }^{24}$ As expected, absolute percentage changes of $\lambda_{\|}$and $\mathrm{K}_{\|}$of the lesionedhemisphere CST were smaller than the absolute percentage changes of $\lambda_{\|}$and $\mathrm{K}_{\|}$of the core infarct, respectively $58 \%$ and $-32 \%$, in a previous study. ${ }^{24}$

The pathologic correlates of increased MK in acute infarct and the downstream effect along the affected WM tracts are still incompletely understood. Acute infarct leading to early CST Wallerian degeneration may result in swelling or beading of downstream axons and dendrites. ${ }^{23-25}$ Alternatively, ischemia could cause increased intra-axonal tortuosity, viscosity changes, microglia upregulation, and mitochondrial or endoplasmatic reticulum changes, which may result in decreased $\mathrm{D}_{\mathrm{a}} \cdot{ }^{22,23,26}$ In agreement with Hui et al, ${ }^{24}$ we found that the decrease in MD of the CST is mainly due to a drop in $\mathrm{D}_{\mathrm{a}}$, consistent with a proposed mechanism of axonal beading, though the CST axial extra-axonal diffusivity was also decreased.

Previous studies have assessed the correlations between DTIderived measures of CST integrity and functional motor impairment in acute stroke. Doughty et $\mathrm{al}^{27}$ identified subtle changes in FA values of the CST in the vicinity of the lesion early after acute ischemic stroke; however, the degree of FA reduction did not significantly improve on the predictive value of the baseline FM-UE scale for 3-month motor outcome. ${ }^{27}$ Similarly, Puig et $\mathrm{al}^{10,28}$ did not find significant correlations between 30 -day motor outcome after stroke and CST FA measured in the pons in the acute phase, specifically $<12$ hours and 3 days poststroke. Feng et $\mathrm{al}^{29}$ developed the weighted CST lesion load, a novel imaging marker obtained by overlaying lesion maps obtained from DWI in the acute phase with a canonical CST. The weighted CST lesion load was found to be a significant predictor of 3-month motor outcome. However, this metric is not a direct measure of CST injury and does not take into account CST pathophysiologic changes after stroke. There is an unfulfilled clinical need for an early imaging marker of CST injury that would complement clinical assessment in the prediction of motor outcome and patient response to rehabilitation therapy. Our work demonstrates that DKI-derived diffusion metrics can detect early microstructural changes of the CST associated with acute stroke. Furthermore, DKI-derived metrics obtained in the acute phase show promising correlations with 3-month motor outcome. If these associations are validated in a future larger prospective study, then these metrics may ultimately prove to be powerful adjuncts to clinical assessment in triaging patients who may benefit from neurorehabilitation treatment.

Stroke rehabilitative treatments are resource-intensive, and patient selection is critical to identify individuals who are likely to benefit from the treatment. ${ }^{30}$ Proposed motor outcome predictors, such as stroke location and volume, ${ }^{31}$ and the degree of CST involvement, ${ }^{6,29}$ may not provide adequate information to predict motor outcome, and a substantial amount of variance remains unexplained. Our results suggest that kurtosis metrics may prove valuable in determining motor pathway integrity. Specifically, we found that elevated $\mathrm{MK} / \mathrm{K}_{\|}$and decreased MD of the CST indicate a poor prognosis, and $\mathrm{MK}$ and $\mathrm{K}_{\|}$had the strongest associations with the 3-month FM-UE scale. Patients with unfavorable diffusion markers may be less likely to benefit from motor rehabilitation programs. Although our preliminary observations will need confirmation in studies with larger cohorts, the use of imaging biomarkers of CST integrity could enable improved selection strategies in future stroke-recovery trials. 
This study has several limitations. We evaluated a small patient cohort, which limits our ability to evaluate the role of diffusion metrics in the context of other prognostic factors. On the other hand, all data were prospectively acquired, and imaging data were obtained with the same MR imaging protocol and scanner. Future studies should include a larger series of patients, which would allow one to determine whether the diffusion metrics are truly independent predictors of motor recovery, over and above the traditional clinical prognostic variables. We did not evaluate the subsequent temporal evolution of the observed CST diffusion measures. Furthermore, we included patients with acute stroke and motor impairment regardless of the vascular territory involved. Our purpose was to evaluate the effects of acute ischemia on CST microstructure regardless of where the core infarct was located along the motor pathways. This study design has been used in previous imaging-based studies on motor outcome after stroke. $^{27,29}$

\section{CONCLUSIONS}

Our preliminary study reveals that early CST microstructural changes immediately following stroke can be detected by using kurtosis metrics, specifically $\mathrm{MK}$ and $\mathrm{K}_{\|}$. We have preliminarily found strong associations between kurtosis metrics obtained at early time points after stroke and long-term motor outcome. A major limitation of this study is the small sample size. This study was designed largely to be one of hypothesis generation, and the usefulness of these imaging markers as independent predictors of disability in the context of other motor outcome predictors must be validated in future studies with larger sample sizes.

\section{ACKNOWLEDGMENTS}

We acknowledge Rachael L. Deardorff for assisting with manuscript preparation.

Disclosures: M. Vittoria Spampinato-RELATED: Grant: South Carolina Translational Research Institute, Comments: National Institutes of Health National Center for Advancing Translational Sciences ULITR001450*. Jens H. Jensen-UNRELATED: Patents (Planned, Pending or Issued): US patent 8811706, issued August 19, 2014, Comments: This is a patent on the DKI technology used in our study. To date, I have not received any royalties from this patent. Joseph A. Helpern-UNRELATED: Patents (Planned, Pending or Issued): New York University and Siemens, Comments: patent for DKI owned by New York University licensed to Siemens*. Leonardo BonilhaUNRELATED: Grants/Grants Pending: National Institutes of Health, American Heart Association, Comments: 1R01DC014021 (National Institutes of Health/National Institute on Deafness and Other Communication Disorders, Principal Investigator; July 1, 2014, to July 1, 2019), Brain Connectivity Supporting Language Recovery in Aphasia, major goal: to investigate how residual structural connectivity enables recovery from aphasia in individuals with stroke; Principal Investigator in 15SFDRN26030003 (American Heart Association; July 1, 2015, to July 1, 2019), Influence of Pre-Morbid Brain Health on Stroke Recovery; major goal: to investigate how prestroke brain health can affect recovery from stroke with regard to focal neurological deficits and quality of life. Steven Kautz-RELATED: Grant: National Institutes of Health, Comments: P20GM109040.* Paul J. Nietert-RELATED: Grant: National Institutes of Health, Comments: National Center for Advancing Translational Sciences grant No. ULITR001450*. W. Feng—RELATED: Grant: Prediction and Imaging Biomarker for Post-stroke Motor Recovery (American Heart Association: 14SDG1829003). *Money paid to the institution.

\section{REFERENCES}

1. Langhorne P, Coupar F, Pollock A. Motor recovery after stroke: a systematic review. Lancet Neurol 2009;8:741-54 CrossRef Medline

2. Hendricks HT, van Limbeek J, Geurts AC, et al. Motor recovery after stroke: a systematic review of the literature. Arch Phys Med Rehabil 2002;83:1629-37 CrossRef Medline

3. Duncan PW, Goldstein LB, Matchar D, et al. Measurement of motor recovery after stroke: outcome assessment and sample size requirements. Stroke 1992;23:1084-89 CrossRef Medline

4. Prabhakaran S, Zarahn E, Riley C, et al. Inter-individual variability in the capacity for motor recovery after ischemic stroke. Neurorehabil Neural Repair 2008;22:64-71 Medline

5. Ward NS, Newton JM, Swayne OB, et al. Motor system activation after subcortical stroke depends on corticospinal system integrity. Brain 2006;129:809-19 CrossRef Medline

6. Puig J, Pedraza S, Blasco G, et al. Acute damage to the posterior limb of the internal capsule on diffusion tensor tractography as an early imaging predictor of motor outcome after stroke. AJNR Am J Neuroradiol 2011;32:857-63 CrossRef Medline

7. Kumar P, Kathuria P, Nair P, et al. Prediction of upper limb motor recovery after subacute ischemic stroke using diffusion tensor imaging: a systematic review and meta-analysis. J Stroke 2016;18: 50-59 CrossRef Medline

8. Yu C, Zhu C, Zhang Y, et al. A longitudinal diffusion tensor imaging study on Wallerian degeneration of corticospinal tract after motor pathway stroke. Neuroimage 2009;47:451-58 CrossRef Medline

9. Groisser BN, Copen WA, Singhal AB, et al. Corticospinal tract diffusion abnormalities early after stroke predict motor outcome. Neurorehabil Neural Repair 2014;28:751-60 CrossRef Medline

10. Puig J, Pedraza S, Blasco G, et al. Wallerian degeneration in the corticospinal tract evaluated by diffusion tensor imaging correlates with motor deficit $\mathbf{3 0}$ days after middle cerebral artery ischemic stroke. AJNR Am J Neuroradiol 2010;31:1324-30 CrossRef Medline

11. Jensen JH, Helpern JA. MRI quantification of non-Gaussian water diffusion by kurtosis analysis. NMR Biomed 2010;23:698-710 CrossRef Medline

12. Jensen JH, Helpern JA, Tabesh A. Leading non-Gaussian corrections for diffusion orientation distribution function. NMR Biomed 2014; 27:202-11 CrossRef Medline

13. Grinberg F, Ciobanu L, Farrher E, et al. Diffusion kurtosis imaging and log-normal distribution function imaging enhance the visualisation of lesions in animal stroke models. NMR Biomed 2012;25: 1295-304 CrossRef Medline

14. Fieremans E, Jensen JH, Helpern JA. White matter characterization with diffusional kurtosis imaging. Neuroimage 2011;58: 177-88 CrossRef Medline

15. Fugl-Meyer AR, Jaasko L, Leyman I, et al. The post-stroke hemiplegic patient. 1: a method for evaluation of physical performance. Scand J Rehabil Med 1975;7:13-31 Medline

16. Shelton FN, Reding MJ. Effect of lesion location on upper limb motor recovery after stroke. Stroke 2001;32:107-12 CrossRef Medline

17. Tabesh A, Jensen JH, Ardekani BA, et al. Estimation of tensors and tensor-derived measures in diffusional kurtosis imaging. Magnetic Reson Med 2011;65:823-36 CrossRef Medline

18. Yeh FC, Verstynen TD, Wang Y, et al. Deterministic diffusion fiber tracking improved by quantitative anisotropy. PLoS One 2013;8: e80713 CrossRef Medline

19. Wakana S, Caprihan A, Panzenboeck MM, et al. Reproducibility of quantitative tractography methods applied to cerebral white matter. Neuroimage 2007;36:630-44 CrossRef Medline

20. Moulton E, Amor-Sahli M, Perlbarg V, et al. Axial diffusivity of the corona radiata at $\mathbf{2 4}$ hours post-stroke: a new biomarker for motor and global outcome. PLoS One 2015;10:e0142910 CrossRef Medline

21. Bhagat YA, Hussain MS, Stobbe RW, et al. Elevations of diffusion anisotropy are associated with hyper-acute stroke: a serial imaging study. Magn Reson Imaging 2008;26:683-93 CrossRef Medline

22. Cheung JS, Wang E, Lo EH, et al. Stratification of heterogeneous diffusion MRI ischemic lesion with kurtosis imaging: evaluation of mean diffusion and kurtosis MRI mismatch in an animal model of transient focal ischemia. Stroke 2012;43:2252-54 CrossRef Medline

23. Jensen JH, Falangola MF, Hu C, et al. Preliminary observations of 
increased diffusional kurtosis in human brain following recent cerebral infarction. NMR Biomed 2011;24:452-57 CrossRef Medline

24. Hui ES, Fieremans E, Jensen JH, et al. Stroke assessment with diffusional kurtosis imaging. Stroke 2012;43:2968-73 CrossRef Medline

25. Budde MD, Frank JA. Neurite beading is sufficient to decrease the apparent diffusion coefficient after ischemic stroke. Proc Natl Acad Sci U S A 2010;107:14472-77 CrossRef Medline

26. Weber RA, Hui ES, Jensen JH, et al. Diffusional kurtosis and diffusion tensor imaging reveal different time-sensitive strokeinduced microstructural changes. Stroke 2015;46:545-50 CrossRef Medline

27. Doughty C, Wang J, Feng W, et al. Detection and predictive value of fractional anisotropy changes of the corticospinal tract in the acute phase of a stroke. Stroke 2016;47:1520-26 CrossRef Medline

28. Puig J, Blasco G, Daunis-I-Estadella J, et al. Decreased corticospinal tract fractional anisotropy predicts long-term motor outcome after stroke. Stroke 2013;44:2016-18 CrossRef Medline

29. Feng W, Wang J, Chhatbar PY, et al. Corticospinal tract lesion load: an imaging biomarker for stroke motor outcomes. Ann Neurol 2015; 78:860-70 CrossRef Medline

30. Stinear C. Prediction of recovery of motor function after stroke. Lancet Neurol 2010;9:1228-32 CrossRef Medline

31. Chen CL, Tang FT, Chen HC, et al. Brain lesion size and location: effects on motor recovery and functional outcome in stroke patients. Arch Phys Med Rehabil 2000;81:447-52 CrossRef Medline 